\title{
Imaging the Alphavirus Exit Pathway
}

Maria Guadalupe Martinez ${ }^{1}$, Erik-Lee Snapp ${ }^{2,3}$, Geoffrey S. Perumal ${ }^{4}$, Frank P. Macaluso ${ }^{4}$ and Margaret Kielian ${ }^{1}$

${ }^{1}$ Dept of Cell Biology, Albert Einstein College of Medicine, Bronx, NY 10461, USA

${ }^{2}$ Dept of Anatomy and Structural Biology, Albert Einstein College of Medicine, Bronx, NY 10461, USA

${ }^{3}$ Gruss Lipper Biophotonics Center, Albert Einstein College of Medicine, Bronx, NY 10461, USA

${ }^{4}$ Analytical Imaging Facility, Albert Einstein College of Medicine, Bronx, NY 10461, USA

Alphaviruses include important and widely distributed human pathogens such as Chikungunya virus and the encephalitic alphaviruses. No vaccines or antiviral therapies are available for most of these viruses. Alphaviruses are small-enveloped RNA viruses and contain an internal nucleocapsid and an external lattice of the viral E2 and E1 transmembrane proteins. Alphavirus bud from the plasma membrane (PM) but the process and dynamics of alphavirus assembly and budding are poorly understood [1].

Budding of the alphavirus particle requires both the capsid protein and the envelope proteins [2] and involves a one-to-one interaction of the cytoplasmic domain of E2 with a hydrophobic pocket on the capsid protein. Mutations in this critical region of E2 block E2-Cp interaction and inhibit budding [3]. Unlike the case for many less ordered enveloped viruses, structural and biochemical studies indicate that host proteins are strictly excluded from the mature alphavirus envelope [1].

Despite the fact that the structures and interactions of the alphavirus capsid and envelope proteins have been extensively characterized, many fundamental questions about alphavirus budding remain. Earlier electron microscopy data suggest that alphavirus budding takes place at localized PM sites. However, it is not clear how these sites are formed or specialized, which viral proteins are involved in the process of cell proteins exclusion from nascent particles, and what functional roles are played by host and viral proteins during assembly and budding. Such questions are amenable to study using imaging methods.

We generated Sindbis viruses (SINV) with fluorescent protein labels on the E2 envelope protein and exploited them to characterize virus assembly and budding in living cells. These viruses will be referred to as WT-GFP and WT-mCherry. We then used these viruses to study budding using a combination of total internal reflection fluorescence microscopy (TIRFM), confocal microscopy, and correlated light and electron microscopy (CLEM). Our studies demonstrated that during virus infection E2 became enriched in localized patches on the PM and in filopodia-like extensions. These E2-labeled patches and extensions contained all of the viral structural proteins. We used CLEM methods to further characterize the E2 PM patches and extensions, and determine if they were sites of virus particle production. Representative SEM images of cells transfected with WT-mCherry or Y400K-mCherry RNA or mocktransfected are shown in Figure 1. The WT-mCherry-infected cells showed abundant particles across the cell surface, along the cell borders, and on plasma membrane extensions (Fig. 1A-B). Correlation between the fluorescence and SEM images of WT-mCherry-infected cells showed that the fluorescent particles observed on short extensions corresponded to nascent virus particles (Fig. 1C). The size of the particles is consistent with that of alphaviruses ( $~ 70 \mathrm{nM}$ in diameter). In contrast, SEM of cells infected with the non-budding mutant or of the uninfected control cells showed a smooth surface without particles (Fig. 1E-J).

Thus, using CLEM we established that the patches and extensions co-localize with virus budding structures, while light microscopy studies showed that they exclude a freely diffusing PM marker protein. Exclusion of this PM marker required the interaction of the E2 protein with the capsid protein, a critical step in virus budding, and was associated with the immobilization of the envelope proteins on 
the cell surface. Virus infection induced at least two different types of extensions: tubulin negative extensions that are shorter, exclude the PM marker and are completely occupied with viral particles when observed by CLEM, and longer extensions that are positive for tubulin staining, appear to mediate virus particle transfer and whose number is reduced in the non-budding mutant SINV. Together our data support a model in which alphavirus budding occurs at specialized sites of virus-induced reorganization of the PM and cytoskeleton.

\section{References:}

[1] Kuhn RJ (2007) Togaviridae: The Viruses and Their Replication. In: Knipe DM, Howley PM, editors. Fields Virology. Fifth ed. Philadelphia, PA: Lippincott, Williams and Wilkins. pp. 1001-1022. [2] Garoff H, Hewson R, Opstelten D-JE (1998) Virus maturation by budding. Microbiol and MolBiolRev 62: 1171-1190.

[3] Zhao H, Lindqvist B, Garoff H, von Bonsdorff C-H, Liljeström P (1994) A tyrosine-based motif in the cytoplasmic domain of the alphavirus envelope protein is essential for budding. EMBO J 13: 42044211.

[4] The authors acknowledge funding to M.K. from the National Institute of General Medical Sciences (GM-057454) and by Cancer Center Core support grant NIH/NCI P30-CA13330.

A
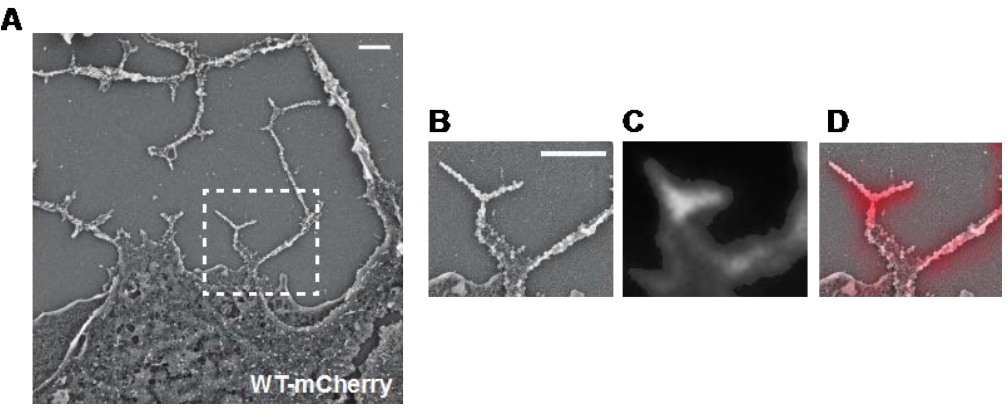

E
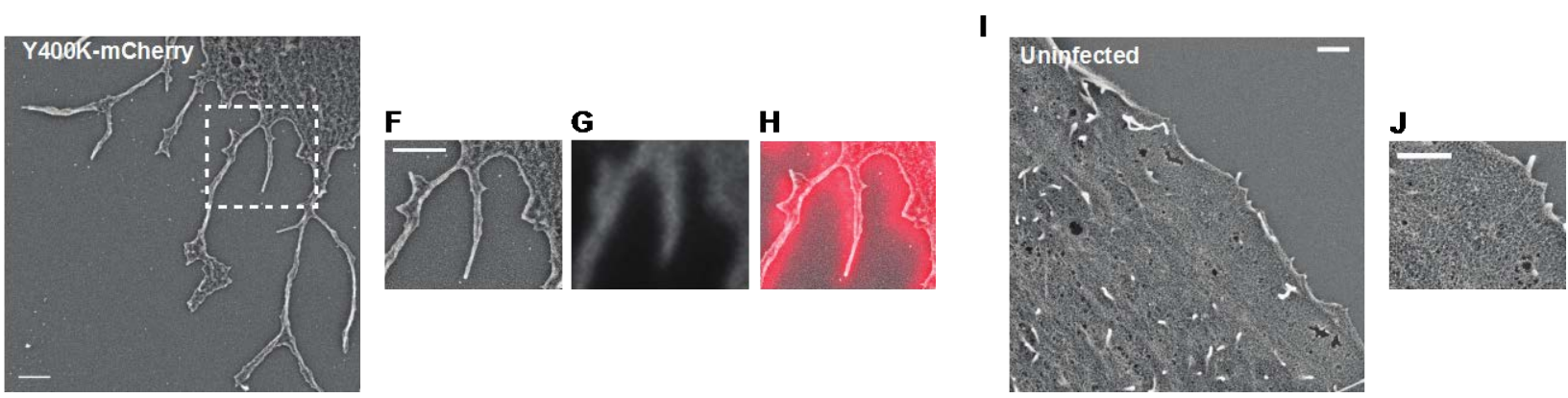

Figure 1. Correlation of scanning electron microscopy (SEM) and fluorescence microscopy shows that E2 fluorescent extensions correlate to budding particles at the cell surface. Vero cells were transfected with WTmCherry (A), Y400K-mCherry RNA (E) or mock transfected (I) and incubated at $37^{\circ} \mathrm{C}$ for $6 \mathrm{~h}$. Transfection media was replaced and after 3h cells were light fixed. Samples were imaged using a Zeiss AxioObserver microscope with "shuffle and find" software. Cells were then processed for SEM and imaged using a Zeiss Supra 40 Field Emission SEM. In the left panels the SEM image of a cell infected with WT-mCherry (A), Y400KmCherry (E) or mock infected (I) are shown (6000X). On the right panels (B,F,J) an inset of the region demarcated with the dashed black box overlaying with the fluorescence of the E2-mCherry proteins is shown $(10000 \mathrm{X})(\mathrm{bar}=1 \mu \mathrm{m})$. 